\title{
A stronger inequality of Cîrtoaje's one with power exponential functions
}

Mitsuhiro Miyagi, Yusuke Nishizawa*

General Education, Ube National College of Technology, Tokiwadai 2-14-1, Ube, Yamaguchi 755-8555, Japan.

Communicated by R. Saadati

\begin{abstract}
In this paper, we will show that $a^{2 b}+b^{2 a}+r(a b(a-b))^{2} \leq 1$ holds for all $0 \leq a$ and $0 \leq b$ with $a+b=1$ and all $0 \leq r \leq 1 / 2$. This gives the first example of a stronger inequality of $a^{2 b}+b^{2 a} \leq 1$. (C) 2015 All rights reserved.
\end{abstract}

Keywords: Power-exponential function, monotonically decreasing function, monotonically increasing function.

2010 MSC: :26D10.

\section{Introduction}

The study of inequalities with power exponential functions is one of the active areas of research in the mathematical analysis. V. Cîrtoaje et al. [1, 2, 3, 4, 5, 6] studied some inequalities with power exponential functions. These problems of inequalities are very simple formula, but these proof are not as simple as it seems. It is noted that

$$
a^{2 b}+b^{2 a}+\left(\frac{a-b}{2}\right)^{2} \leq 2
$$

and

$$
a^{3 b}+b^{3 a}+\left(\frac{a-b}{2}\right)^{4} \leq 2
$$

holds for all $0 \leq a$ and $0 \leq b$ with $a+b=2$. These inequalities (1.1) and (1.2) are proved by V. Cîrtoaje et al. [2, 6, respectively. In this paper, we will show that

$$
a^{2 b}+b^{2 a}+r(a b(a-b))^{2} \leq 1
$$

\footnotetext{
* Corresponding author

Email addresses: miyagi@ube-k.ac.jp (Mitsuhiro Miyagi), yusuke@ube-k.ac.jp (Yusuke Nishizawa)
} 
holds for all $0 \leq a$ and $0 \leq b$ with $a+b=1$ and all $0 \leq r \leq 1 / 2$, which is a stronger inequality of

$$
a^{2 b}+b^{2 a} \leq 1 .
$$

The above inequality (1.4) is Conjecture 4.8 in [2] and proved by V. Cîrtoaje [3]. The following is our main theorem.

Theorem 1.1. For all $0 \leq a$ and $0 \leq b$ with $a+b=1$ and all $0 \leq r \leq 1 / 2$, the inequality (1.3) holds.

This gives the first example of a stronger inequality of 1.4 .

\section{Proof of Theorem 1.1}

Proof. Without loss of generically, we assume that

$$
0 \leq b \leq \frac{1}{2} \leq a \leq 1
$$

Applying Lemma 7.1 in [3], we have

$$
a^{2 b} \leq 1-4 a b^{2}-2 a b(a-b) \ln a
$$

and since the inequality (1.3) is strictly increasing for $0 \leq r \leq 1 / 2$, it suffices to show that

$$
b^{2 a}+\frac{1}{2}(a b(a-b))^{2} \leq 4 a b^{2}+2 a b(a-b) \ln a .
$$

We assume that $a=(1+t) / 2$ and $b=(1-t) / 2$, where $0 \leq t \leq 1$. Here, the inequality (2.1) is equivalent to

$$
\left(\frac{1-t}{2}\right)^{t+1}+\frac{1}{32}(-1+t)^{2}(1+t)\left(-16+t^{2}+t^{3}\right)+\frac{1}{2}(1-t) t(1+t)(\ln (1+t)-\ln 2) \leq 0
$$

Moreover, from Lemma 2.1 in [6], we have

$$
(1-t)^{1+t} \leq \frac{1}{4}(1-t)^{2}\left(2-t^{2}\right)\left(2+2 t+t^{2}\right)
$$

and by the well known fact we have

$$
\begin{aligned}
2^{-t} & =e^{-t \ln 2} \\
& =1-(\ln 2) t+\frac{((\ln 2) t)^{2}}{2}-\frac{((\ln 2) t)^{3}}{3 !}+\frac{((\ln 2) t)^{4}}{4 !}-\cdots \\
& \leq 1-(\ln 2) t+\frac{((\ln 2) t)^{2}}{2}-\frac{((\ln 2) t)^{3}}{3 !}+\frac{((\ln 2) t)^{4}}{4 !} .
\end{aligned}
$$

Therefore, it suffices to show that

$$
\begin{aligned}
F(t):=\frac{1}{2} & \left(1-(\ln 2) t+\frac{((\ln 2) t)^{2}}{2}-\frac{((\ln 2) t)^{3}}{3 !}+\frac{((\ln 2) t)^{4}}{4 !}\right) \\
& \times \frac{1}{4}(1-t)^{2}\left(2-t^{2}\right)\left(2+2 t+t^{2}\right)+\frac{1}{32}(-1+t)^{2}(1+t)\left(-16+t^{2}+t^{3}\right) \\
& +\frac{1}{2}(1-t) t(1+t)(\ln (1+t)-\ln 2) \leq 0 .
\end{aligned}
$$

We have the fourth derivated function

$$
F^{(4)}(t)=\frac{d^{4}}{d t^{4}} F(t)=\frac{f(t)}{(t+1)^{3}}
$$


of $F(t)$, where

$$
\begin{aligned}
f(t)= & 62+126 t-33 t^{2}-375 t^{3}-405 t^{4}-135 t^{5} \\
& +12(1+t)^{3}\left(-2-15 t+35 t^{3}\right)(\ln 2) \\
& -6(1+t)^{3}\left(4-10 t-45 t^{2}+70 t^{4}\right)(\ln 2)^{2} \\
& +2(1+t)^{3}\left(4+20 t-30 t^{2}-105 t^{3}+126 t^{5}\right)(\ln 2)^{3} \\
& -(1+t)^{3}\left(-2+10 t+30 t^{2}-35 t^{3}-105 t^{4}+105 t^{6}\right)(\ln 2)^{4} .
\end{aligned}
$$

Then, we have derivatives

$$
\begin{aligned}
f^{(6)}(t)= & -5040\left(-60-78(\ln 2)^{2}-756 t(\ln 2)^{2}-1008 t^{2}(\ln 2)^{2}-35(\ln 2)^{3}\right. \\
& \left.+180(\ln 2)+420 t(\ln 2)+210 t(\ln 2)^{3}+1260 t^{2}(\ln 2)^{3}+1260 t^{3}(\ln 2)^{3}\right)
\end{aligned}
$$

Since

we have

$$
\frac{69}{100}<\ln 2<\frac{7}{10}
$$

$$
\begin{aligned}
& -60-78(\ln 2)^{2}-756 t(\ln 2)^{2}-1008 t^{2}(\ln 2)^{2}-35(\ln 2)^{3} \\
& +180(\ln 2)+420 t(\ln 2)+210 t(\ln 2)^{3}+1260 t^{2}(\ln 2)^{3}+1260 t^{3}(\ln 2)^{3} \\
& >-60-78\left(\frac{7}{10}\right)^{2}-756 t\left(\frac{7}{10}\right)^{2}-1008 t^{2}\left(\frac{7}{10}\right)^{2}-35\left(\frac{7}{10}\right)^{3} \\
& +180\left(\frac{69}{100}\right)+420 t\left(\frac{69}{100}\right)+210 t\left(\frac{69}{100}\right)^{3}+1260 t^{2}\left(\frac{69}{100}\right)^{3}+1260 t^{3}\left(\frac{69}{100}\right)^{3} \\
& =\frac{1}{100000}\left(1397500-1165311 t-7999866 t^{2}+41392134 t^{3}\right) \\
& >\frac{1}{100000}\left(1300000-1200000 t-8000000 t^{2}+40000000 t^{3}\right) \\
& =13-12 t-80 t^{2}+400 t^{3} .
\end{aligned}
$$

We set

$$
\tilde{f}(t)=13-12 t-80 t^{2}+400 t^{3}
$$

then we have

$$
\tilde{f}^{\prime}(t)=4\left(-3-40 t+300 t^{2}\right)
$$

Since

$$
\tilde{f}^{\prime}\left(\frac{2-\sqrt{13}}{30}\right)=0 \quad \text { and } \quad \tilde{f}^{\prime}\left(\frac{2+\sqrt{13}}{30}\right)=0 \text {, }
$$

we have

$$
\tilde{f}(t) \geq \tilde{f}\left(\frac{2+\sqrt{13}}{30}\right) \cong 10.5742 .
$$

Hence, we can get

$$
f^{(6)}(t)<0
$$

Thus, $f^{(5)}(t)$ is strictly decreasing for $0<t<1$. We have

$$
\begin{aligned}
f^{(5)}(t) & =-16200+151200(1+2 t)(\ln 2) \\
& -10800\left(11+84 t+98 t^{2}\right)(\ln 2)^{2} \\
& +720\left(-73+546 t+2646 t^{2}+2352 t^{3}\right)(\ln 2)^{3} \\
& -3600\left(-13-49 t+147 t^{2}+588 t^{3}+441 t^{4}\right)(\ln 2)^{4},
\end{aligned}
$$




$$
\begin{aligned}
f^{(5)}(0) & =-16200+151200(\ln 2)-118800(\ln 2)^{2}-52560(\ln 2)^{3}+46800(\ln 2)^{4} \\
& \cong 24825.3
\end{aligned}
$$

and

$$
\begin{aligned}
f^{(5)}(1) & =-16200+453600(\ln 2)-2084400(\ln 2)^{2}+3939120(\ln 2)^{3}-4010400(\ln 2)^{4} \\
& \cong-317162 .
\end{aligned}
$$

Since $f^{(5)}(t)$ is strictly decreasing for $0<t<1$, there exists uniquely a real number $0<t_{1}<1$ such that $f^{(5)}\left(t_{1}\right)=0$. Since $f^{(5)}(t)>0$ for $0<t<t_{1}$ and $f^{(5)}(t)<0$ for $t_{1}<t<1, f^{(4)}(t)$ is strictly increasing for $0<t<t_{1}$ and $f^{(4)}(t)$ is strictly decreasing for $t_{1}<t<1$. We have

$$
\begin{aligned}
f^{(4)}(t) & =-9720-16200 t \\
& +4320\left(6+35 t+35 t^{2}\right)(\ln 2) \\
& -3600\left(-3+33 t+126 t^{2}+98 t^{3}\right)(\ln 2)^{2} \\
& +240\left(-77-219 t+819 t^{2}+2646 t^{3}+1764 t^{4}\right)(\ln 2)^{3} \\
& -120\left(-22-390 t-735 t^{2}+1470 t^{3}+4410 t^{4}+2646 t^{5}\right)(\ln 2)^{4}, \\
f^{(4)}(0)= & -9720+25920(\ln 2)+10800(\ln 2)^{2}-18480(\ln 2)^{3}+2640(\ln 2)^{4} \\
\cong & 7890.38
\end{aligned}
$$

and

$$
\begin{aligned}
f^{(4)}(1) & =-25920+328320(\ln 2)-914400(\ln 2)^{2}+1183920(\ln 2)^{3}-885480(\ln 2)^{4} \\
& \cong-47797.5
\end{aligned}
$$

Since $f^{(4)}(t)$ is strictly increasing for $0<t<t_{1}$ and $f^{(4)}(t)$ is strictly decreasing for $t_{1}<t<1$, there exists uniquely a real number $t_{1}<t_{2}<1$ such that $f^{(4)}\left(t_{2}\right)=0$. Since $f^{(4)}(t)>0$ for $0<t<t_{2}$ and $f^{(4)}(t)<0$ for $t_{2}<t<1, f^{(3)}(t)$ is strictly increasing for $0<t<t_{2}$ and $f^{(3)}(t)$ is strictly decreasing for $t_{2}<t<1$. We have

$$
\begin{aligned}
f^{(3)}(t) & =-2250-9720 t-8100 t^{2} \\
& +144\left(-6+180 t+525 t^{2}+350 t^{3}\right)(\ln 2) \\
& -36\left(-161-300 t+1650 t^{2}+4200 t^{3}+2450 t^{4}\right)(\ln 2)^{2} \\
& +12\left(-131-1540 t-2190 t^{2}+5460 t^{3}+13230 t^{4}+7056 t^{5}\right)(\ln 2)^{3} \\
& -6\left(83-440 t-3900 t^{2}-4900 t^{3}+7350 t^{4}+17640 t^{5}+8820 t^{6}\right)(\ln 2)^{4} \\
f^{(3)}(0) & =-2250-864(\ln 2)+5796(\ln 2)^{2}-1572(\ln 2)^{3}-498(\ln 2)^{4} \\
& \cong-702.644
\end{aligned}
$$

and

$$
\begin{aligned}
f^{(3)}(1) & =-20070+151056(\ln 2)-282204(\ln 2)^{2}+262620(\ln 2)^{3}-147918(\ln 2)^{4} \\
& \cong 2362.55 .
\end{aligned}
$$

Since $f^{(3)}(t)$ is strictly decreasing for $0<t<t_{2}$ and $f^{(3)}(t)$ is strictly decreasing for $t_{2}<t<1$, there exists uniquely a real number $0<t_{3}<t_{2}$ such that $f^{(3)}\left(t_{3}\right)=0$. Since $f^{(3)}(t)<0$ for $0<t<t_{3}$ and $f^{(3)}(t)>0$ 
for $t_{3}<t<1, f^{(2)}(t)$ is strictly decreasing for $0<t<t_{3}$ and $f^{(2)}(t)$ is strictly increasing for $t_{3}<t<1$. We have

$$
\begin{aligned}
f^{(2)}(t) & =-66-2250 t-4860 t^{2}-2700 t^{3} \\
& +72(1+t)\left(-17+5 t+175 t^{2}+175 t^{3}\right)(\ln 2) \\
& -36(1+t)\left(-21-140 t-10 t^{2}+560 t^{3}+490 t^{4}\right)(\ln 2)^{2} \\
& +12(1+t)\left(14-145 t-625 t^{2}-105 t^{3}+1470 t^{4}+1176 t^{5}\right)(\ln 2)^{3} \\
& -6(1+t)\left(18+65 t-285 t^{2}-1015 t^{3}-210 t^{4}+1680 t^{5}+1260 t^{6}\right)(\ln 2)^{4} \\
& \quad f^{(2)}(0)=-66-1224(\ln 2)+756(\ln 2)^{2}+168(\ln 2)^{3}-108(\ln 2)^{4} \\
& \cong-520.172
\end{aligned}
$$

and

$$
\begin{aligned}
f^{(2)}(1) & =-9876+48672(\ln 2)-63288(\ln 2)^{2}+42840(\ln 2)^{3}-18156(\ln 2)^{4} \\
& \cong 3529.68
\end{aligned}
$$

Since $f^{(2)}(t)$ is strictly decreasing for $0<t<t_{3}$ and $f^{(2)}(t)$ is strictly increasing for $t_{3}<t<1$, there exists uniquely a real number $t_{3}<t_{4}<1$ such that $f^{(2)}\left(t_{4}\right)=0$. Since $f^{(2)}(t)<0$ for $0<t<t_{4}$ and $f^{(2)}(t)>0$ for $t_{4}<t<1, f^{\prime}(t)$ is strictly decreasing for $0<t<t_{4}$ and $f^{\prime}(t)$ is strictly increasing for $t_{4}<t<1$. We have

$$
\begin{aligned}
f^{\prime}(t) & =126-66 t-1125 t^{2}-1620 t^{3}-675 t^{4} \\
+ & 36(1+t)^{2}\left(-7-20 t+35 t^{2}+70 t^{3}\right)(\ln 2) \\
- & 6(1+t)^{2}\left(2-130 t-225 t^{2}+280 t^{3}+490 t^{4}\right)(\ln 2)^{2} \\
+ & 2(1+t)^{2}\left(32+20 t-465 t^{2}-630 t^{3}+630 t^{4}+1008 t^{5}\right)(\ln 2)^{3} \\
- & (1+t)^{2}\left(4+100 t+45 t^{2}-630 t^{3}-735 t^{4}+630 t^{5}+945 t^{6}\right)(\ln 2)^{4} \\
& f^{\prime}(0)=126-252(\ln 2)-12(\ln 2)^{2}+64(\ln 2)^{3}-4(\ln 2)^{4} \\
& \cong-34.0483
\end{aligned}
$$

and

$$
\begin{aligned}
f^{\prime}(1) & =-3360+11232(\ln 2)-10008(\ln 2)^{2}+4760(\ln 2)^{3}-1436(\ln 2)^{4} \\
& \cong 870.774 .
\end{aligned}
$$

Since $f^{\prime}(t)$ is strictly decreasing for $0<t<t_{4}$ and $f^{\prime}(t)$ is strictly increasing for $t_{4}<t<1$, there exists uniquely a real number $t_{4}<t_{5}<1$ such that $f^{\prime}\left(t_{5}\right)=0$. Since, $f^{\prime}(t)<0$ for $0<t<t_{5}$ and $f^{\prime}(t)>0$ for $t_{5}<t<1, f(t)$ is strictly decreasing for $0<t<t_{5}$ and $f(t)$ is strictly increasing for $t_{5}<t<1$. Since

$$
\begin{gathered}
f(0)=2\left(31-12(\ln 2)-12(\ln 2)^{2}+4(\ln 2)^{3}+(\ln 2)^{4}\right) \cong 36.9595, \\
f(1)=-8\left(95-216(\ln 2)+114(\ln 2)^{2}-30(\ln 2)^{3}+3(\ln 2)^{4}\right) \cong 73.9711,
\end{gathered}
$$

and

$$
\begin{aligned}
f\left(\frac{1}{2}\right) & =\frac{1}{512}\left(20656-106272(\ln 2)+81648(\ln 2)^{2}-9288(\ln 2)^{3}-2079(\ln 2)^{4}\right) \\
& \cong-33.889
\end{aligned}
$$


Since $f(t)$ is strictly decreasing for $0<t<t_{5}$ and $f(t)$ is strictly increasing for $t_{5}<t<1$, we have only two real numbers $a_{1}$ and $a_{2}$ with $0<a_{1}<1 / 2<a_{2}<1$ such that $f\left(a_{1}\right)=0$ and $f\left(a_{2}\right)=0$. Since $f(t)>0$ for all $0<t<a_{1}, a_{2}<t<1$ and $f(t)<0$ for all $a_{1}<t<a_{2}, F^{(3)}(t)$ is strictly increasing for $0<t<a_{1}$, $a_{2}<t<1$ and $F^{(3)}(t)$ is strictly decreasing for $a_{1}<t<a_{2}$. We have

$$
F^{(3)}(t)=\frac{g(t)}{(t+1)^{2}}
$$

where

$$
\begin{aligned}
g(t)= & 200 t+304 t^{2}-60 t^{3}-360 t^{4}-180 t^{5} \\
& +12 t(1+t)^{2}\left(-8-30 t+35 t^{3}\right)(\ln 2) \\
& -24(1+t)^{2}\left(1+4 t-5 t^{2}-15 t^{3}+14 t^{5}\right)(\ln 2)^{2} \\
& +2(1+t)^{2}\left(-4+16 t+40 t^{2}-40 t^{3}-105 t^{4}+84 t^{6}\right)(\ln 2)^{3} \\
& -t(1+t)^{2}\left(-8+20 t+40 t^{2}-35 t^{3}-84 t^{4}+60 t^{6}\right)(\ln 2)^{4} \\
& +48(1+t)^{2} \ln (1+t) .
\end{aligned}
$$

We have

$$
\begin{gathered}
F^{(3)}(0)=-\frac{1}{2}(\ln 2)^{2}(3+\ln 2) \cong-0.887192 \\
F^{(3)}(1)=\frac{1}{16}\left(-24+12(\ln 2)+24(\ln 2)^{2}-18(\ln 2)^{3}+7(\ln 2)^{4}\right) \cong-0.533122
\end{gathered}
$$

and

$$
\begin{aligned}
F^{(3)}\left(\frac{1}{2}\right)=\frac{1}{4608} & \left(17968-46008(\ln 2)-2160(\ln 2)^{2}\right. \\
+ & \left.2160(\ln 2)^{3}-477(\ln 2)^{4}+13824(\ln 3)\right) \cong 0.181499
\end{aligned}
$$

Since we have only two real numbers $a_{3}$ and $a_{4}$ with $0<a_{3}<1 / 2$ and $1 / 2<a_{4}<1$ such that $F^{(3)}\left(a_{3}\right)=0$ and $F^{(3)}\left(a_{4}\right)=0, F^{(3)}(t)<0$ for all $0<t<a_{3}, a_{4}<t<1$ and $F^{(3)}(t)>0$ for all $a_{3}<t<a_{4}$. Therefore, $F^{(2)}(t)$ is strictly decreasing for $0<t<a_{3}, a_{4}<t<1$ and $F^{(2)}(t)$ is strictly increasings for $a_{3}<t<a_{4}$. We have

$$
F^{(2)}(t)=\frac{h(t)}{96(t+1)}
$$

where

$$
\begin{aligned}
h(t)= & -6\left(15+15 t-76 t^{2}-60 t^{3}+45 t^{4}+45 t^{5}\right) \\
& +24(1+t)\left(4-12 t^{2}-30 t^{3}+21 t^{5}\right)(\ln 2) \\
& -12(1+t)\left(-4+12 t+24 t^{2}-20 t^{3}-45 t^{4}+28 t^{6}\right)(\ln 2)^{2} \\
& +4 t(1+t)\left(-12+24 t+40 t^{2}-30 t^{3}-63 t^{4}+36 t^{6}\right)(\ln 2)^{3} \\
& -t^{2}(1+t)\left(-24+40 t+60 t^{2}-42 t^{3}-84 t^{4}+45 t^{6}\right)(\ln 2)^{4} \\
& +288 t(1+t) \ln (1+t) .
\end{aligned}
$$

We have

$$
\begin{gathered}
F^{(2)}(0)=\frac{1}{16}\left(-15+16(\ln 2)+8(\ln 2)^{2}\right) \cong-0.00412631 \\
F^{(2)}(1)=\frac{1}{96}\left(48-120(\ln 2)+60(\ln 2)^{2}-20(\ln 2)^{3}+5(\ln 2)^{4}\right) \cong-0.123508
\end{gathered}
$$


and

$$
\begin{aligned}
F^{(2)}\left(\frac{1}{2}\right)=\frac{1}{24576} & \left(-224-49728(\ln 2)-9600(\ln 2)^{2}\right. \\
& \left.+1472(\ln 2)^{3}-77(\ln 2)^{4}+36864(\ln 3)\right) \cong 0.0678104 .
\end{aligned}
$$

Since we have only two real numbers $a_{5}$ and $a_{6}$ with $0<a_{5}<1 / 2$ and $1 / 2<a_{6}<1$ such that $F^{(2)}\left(a_{5}\right)=0$ and $F^{(2)}\left(a_{6}\right)=0, F^{(2)}(t)<0$ for all $0<t<a_{5}, a_{6}<t<1$ and $F^{(2)}(t)>0$ for all $a_{5}<t<a_{6}$. Therefore, $F^{\prime}(t)$ is strictly decreasing for $0<t<a_{5}, a_{6}<t<1$ and $F^{\prime}(t)$ is strictly increasing for $a_{5}<t<a_{6}$. We have

$$
F^{\prime}(t)=\frac{p(t)}{96}
$$

where

$$
\begin{aligned}
p(t)= & -6(-1+t)^{2} t\left(7+18 t+9 t^{2}\right) \\
& +12 t\left(8-8 t^{2}-15 t^{3}+7 t^{5}\right)(\ln 2) \\
& -12(-1+t) t\left(4-2 t-10 t^{2}-5 t^{3}+4 t^{4}+4 t^{5}\right)(\ln 2)^{2} \\
& +2(-1+t) t^{2}\left(12-4 t-24 t^{2}-12 t^{3}+9 t^{4}+9 t^{5}\right)(\ln 2)^{3} \\
& -(-1+t) t^{3}\left(8-2 t-14 t^{2}-7 t^{3}+5 t^{4}+5 t^{5}\right)(\ln 2)^{4} \\
& +48\left(-1+3 t^{2}\right) \ln (1+t)
\end{aligned}
$$

We have

$$
F^{\prime}(0)=0
$$

and

$$
F^{\prime}(1)=0 .
$$

Since there exists uniquely a real number $a_{7}$ with $0<a_{7}<1$ such that $F^{\prime}\left(a_{7}\right)=0, F(t)$ is strictly decreasing for $0<t<a_{7}$ and $F(t)$ is strictly increasing for $a_{7}<t<1$ Hence, we can get

$$
F(t) \leq \max \{F(0), F(1)\} .
$$

Since $F(0)=F(1)=0$, we have $F(t) \leq 0$ for all $0 \leq t \leq 1$. Therefore, the proof of Theorem 1.1 is completed.

Problem 2.1. What is the maximum value of a nonnegative real number $r$ in the inequality $a^{2 b}+b^{2 a}+$ $r(a b(a-b))^{2} \leq 1$ for all nonnegative real numbers $a$ and $b$ with $a+b=1$ ?

\section{References}

[1] A. Coronel, F. Huancas On the inequality $a^{2 a}+b^{2 b}+c^{2 c} \geq a^{2 b}+b^{2 c}+c^{2 a}$, Aust. J. Math. Anal. Appl., 9 (2012), 5 pages. 1

[2] V. Cîrtoaje, On some inequalities with power-exponential functions, JIPAM. J. Inequal. Pure Appl. Math., 10 (2009), 6 pages. 1, 1, 1

[3] V. Cîrtoaje, Proofs of three open inequalities with power-exponential functions, J. Nonlinear Sci. Appl., 4 (2011), 130-137. 1, 1,2

[4] L. Matejicka, Proof of one open inequality, J. Nonlinear Sci. Appl., 7 (2014), 51-62. 1

[5] M. Miyagi, Y. Nishizawa, Proof of an open inequality with double power-exponential functions, J. Inequal. Appl., 2013 (2013), 11 pages. 1

[6] M. Miyagi, Y. Nishizawa, A short proof of an open inequality with power-exponential functions, Aust. J. Math. Anal. Appl., 11 (2014), 3 pages. 